GU J Sci, Part C, 7(1): 203-212 (2019)

Gazi Üniversitesi
Fen Bilimleri Dergisi
PART C: TASARIM VE TEKNOLOJI
http://dergipark.gov.tr/gujsc

\title{
Karma Weibull/Log-Normal Sönümlenmeli Kanalın Hata Olasılığı Analizi
}

\author{
Mehmet BILIMiM ${ }^{1 *}$ \\ ${ }^{I}$ Nuh Naci Yazgan Üniversitesi, Mühendislik Fakültesi, Elektrik-Elektronik Mühendisliği Bölümü, Kocasinan/KAYSERİ
}

Öz

Makale Bilgisi

Başvuru: 06/09/2018

Düzeltme: 03/01/2019

Kabul: 14/01/2019

\begin{tabular}{l} 
Anahtar Kelimeler \\
\hline $\begin{array}{l}\text { Weibull/log-normal, } \\
\text { hata olastlığl, } \\
\text { kümülatif dağıllm } \\
\text { fonksiyonu }\end{array}$ \\
Keywords \\
\hline $\begin{array}{l}\text { Weibull/log-normal, } \\
\text { error probability, } \\
\text { cumulative distribution } \\
\text { function. }\end{array}$
\end{tabular}

\begin{abstract}
$\mathrm{Bu}$ çalışmada, tek-girişli tek-çıkışlı (single-input single-output, SISO) sistemin karma Weibull/log-normal sönümlenmeli kanallardaki hata olasılığ incelenmiştir. Ele alınan SISO sisteminin birçok gerçekçi iletişim senaryosundaki sönümlenme etkilerini karakterize etmede faydalı olan karma Weibull/log-normal ortamında analizinin yapılması hedeflenmiştir. Bu yüzden, Weibull/log-normal sönümlenmeli kanalının kümülatif dağılım fonksiyonu (cumulative distribution function, CDF) kullanılarak hata olasılığı ifadesi kapalı formda elde edilmiştir. Önerilen bu ifade CDF tabanlı olup, farklı ikili sayısal modülasyon türleri için geçerlidir. Çalışmada sunulan bütün sonuçlar bilgisayar benzetimleri ile doğrulanmıştır.
\end{abstract}

\section{Error Probability Analysis of a Composite Weibull/Log-Normal Fading Channel}

\begin{abstract}
In this study, error probability of a single-input single-output (SISO) system is investigated over a composite Weibull/log-normal fading channel. We aim to study a SISO system in the composite Weibull/log-normal composite fading model which is useful in characterizing fading effects in numerous realistic communication scenarios. Therefore, a closed-form error probability expression was derived by using cumulative distribution function (CDF) of a Weibull/log-normal fading channel. The proposed error probability expression is based on CDF and is valid for different binary digital modulation types. Results that are given in this study were confirmed by computer simulations.
\end{abstract}

\section{GİRIŞ (INTRODUCTION)}

Gelecek nesil kablosuz haberleşme sistemlerinin oluşturulmasında yüksek veri hızı, düşük güç tüketimi, düşük gecikme zamanı (latency), yüksek spektral ve enerji verimliliği gibi faktörler gözetilmektedir [1-5]. Bütün bu faktörlere ek olarak, farklı türdeki haberleşme sistemlerinin analizleri gerçekleştirilirken kablosuz iletim ortamlarının tanımlanması da önemli bir husus olarak ön plana çıkmaktadır. Kablosuz haberleşme sistemleri için en yaygın olarak kullanılan başlıca sönümlü kanal modelleri: Rayleigh, Rician ve Nakagami$m$ olarak sıralanmaktadır. Rayleigh kanal modeli, verici ile alıcı arasındaki direk görüş hattının olmadığı (non line-of-sight, NLOS) durumu temsil etmekte iken Rician kanal modeli ise verici ve alıcı arasındaki direk görüş hattının olduğu (line-of-sight, LOS) durumu tanımlamaktadır. Küçük ölçekli sönümlenme ve bina dışı ortamlardaki iletim hattının tanımlanmasında oldukça başarılı olan bir diğer iletim kanalı modeli ise Nakagami- $m$ dağılımıdır [6]. Bu kanal modelinde $m$ sönümlenme parametresinin ayarlanması ile kanal ortamının sönümlenme şiddeti artmakta veya azalmaktadır.

Bütün bu anlatılanların yansıra, kablosuz iletim ortamları çok-yollu sönümlenme ve gölgeleme etkileri gibi bozucu durumlardan etkilenmektedir [7]. Bu sebeple daha gerçekçi kanal ortamlarının temsil edilmesi için bu bozucu etkileri ele alan kanal tipleri ortaya atılmıştır [8-13]. [8]-[10]'da sunulan çalışmalarda sırasıyla, daha önce bahsedilen klasik sönümlenme modellerini kapsayan $\kappa-\mu$, gölgeli $\kappa-\mu$ ve $\alpha-\mu$ sönümlü kanal modelleri sunulmuştur. $\kappa-\mu$ kanal dağılımının fiziksel ölçümlerde küçük ölçekli sönümlenmeyi daha iyi temsil ettiği ve bu kanal tipinin LOS durumunu çok-yollu sönümlenme etkisi altında daha iyi tanımladığ 
gösterilmiştir [9]. [10]'daki çalışmada ise [9]'da sunulan $\kappa-\mu$ kanal tipine gölgeleme etkisi de dahil edilerek daha farklı bir kanal dağılımı önerilmiştir. Yacoub, [10]'da yaptığı çalışmada $\alpha-\mu$ olarak adlandırılan ve deneysel ölçümlerde bina dışı çevresel etkileri göz önüne alan farklı bir kanal tipi geliştirmiştir. Küçük ölçekli sönümlenme etkisi ile birlikte NLOS durumunun da var olduğu kablosuz iletim ortamının tanımlanması için $\eta-\mu$ kanal dağılımı ortaya atılmıştır [11]. Buna ilaveten [12]'de sunulan çalışmada iki değişkenli $\kappa-\mu$ dağılımına sahip farklı bir kanal modeli geliştirilmiştir. Son dönemde yapılan [13] çalışmada ise $\alpha-\eta-\kappa-\mu$ isimli çok daha komplike ve genelleştirilmiş bir kanal modeli önerilmiştir. Ancak bu kanal tipi ise matematiksel olarak çok karmaşıktır.

Bütün bu kanal modellerine [8-13] ek olarak, Weibull sönümlü kanal modeli [14, 15]'te sunulan çalışmalarda önerilmiş, bina içi ve dışı ortamlarda çok yollu yayılma etkisini yansıtabilme özellikleri deneysel olarak gösterilmiştir. Weibull dağılımı [8-13]'te sunulan kanal tiplerine göre daha az karmaşıklığa sahiptir. Bu kanal modeli farklı kablosuz iletişim uygulamalarında kullanılmıştır [16-19]. Weibull iletim kanalının belirli frekans aralığında çalışan telsiz sayısal sistemler için çok iyi uyum gösterdiği [16]'daki çalışmada detaylı olarak izah edilmiştir. Bilim vd. çöz-ve-aktar işbirlikli serpiştirme bölmeli çoklu erişim sistemlerinin Weibull sönümlü kanallardaki performansının teorik ve simülasyon incelemelerini sunmuşlardır [17, 18]. Bu çalışmalara ek olarak, kablosuz iletim hattının doğru tanımlanabilmesi ve kuramsal çalışmalar için "IEEE Vehicular Technology Society Committee" tarafindan Weibull kanal ortamının kullanılması [19]'da verilen raporda tavsiye edilmektedir. Diğer bir deyişle, [19]'da sunulan raporda, Weibull kanal ortamının gerçek kablosuz iletim ortamları ile test sonuçlarının uygunluğu açıkça izah edilmiştir. Weibull kanalının bütün bu anlatılan özelliklerine ek olarak log-normal dağılımı ile birleşimiyle yeni bir karma tipi olarak Weibull/log-normal sönümlü kanal modeli geliştirilmiştir [20]. Bu sayede log-normal dağılımı kullanılarak gölgeleme etkisinin de Weibull dağılımına yansıtılması hedeflenmiştir. Bu çalışmada da hem bu özellikleri barındıran Weibull kanal modeli hem de gölgeleme etkisinin yansıtılmasındaki başarısı oldukça iyi olan log-normal dağılımının birlikte kullanıldığı karma Weibull/log-normal kanal modeli seçilmiştir.

Bilindiği kadarıyla, literatürde Weibull/log-normal karma sönümlü bir kanalda SISO sisteminin hata olasılığının teorik olarak analizi sunulmamıştır. Literatürde bu noktada önemli bir boşluk bulunmaktadır. Weibull/log-normal karma sönümlü kanal modelinin bina içi ve dışı ortamları temsil etmedeki, çok-yollu ve gölgeleme etkilerini yansıtmadaki kabiliyeti göz önünde bulundurulursa, bir SISO sisteminin bu kanal modelindeki hata olasılığının yapılması literatüre katkı sağlayacaktır. Yapılan teorik analizde öncelikle bir karma Weibull/log-normal sönümlü kanal modelinin kümülatif dağılım fonksiyonu (cumulative distribution function, CDF) ifadesi ele alınmıştır. Daha sonra bu CDF ifadesi kullanılarak hata olasılığı analizi incelenmiştir. Yapılan analiz sonucunda kapalı formda bir hata olasılığı ifadesi türetilmiştir. Türetilen bu ifade farklı sayısal modülasyon türleri için geçerlidir. Elde edilen kapalı formdaki hata olasılığı ifadesi yardımıyla sunulan sonuçlar bilgisayar benzetimleri ile doğrulanmıştır. Sunulan sonuçlarda farklı sönümlenme parametre değerleri ele alınmış ve beklenildiği gibi sönümlenme parametresinin artması ile sonuçların iyileştiği gösterilmiştir. Ayrıca sistemdeki farklı modülasyon türleri seçilerek detaylı analizler sunulmuş̧tur.

\section{SISTEM VE KANAL MODELİ (SYSTEM AND CHANNEL MODEL)}

Bu çalışmada, tek bir kablosuz iletim hattına sahip sönümlü bir kanal ile haberleşen verici ve alıcı çifti düşünülmektedir. Verici ve alıcıdaki modülasyon/demodülasyon türleri: ikili faz kaydırmalı anahtarlama (binary phase shift keying, BPSK), ayrımsal faz kaydırmalı anahtarlama (differential phase shift keying, DPSK) ve ikili frekans kaydırmalı anahtarlama (binary frequency shift keying, BFSK) şeklindedir. Bu çalışmada, daha öncede belirtildiği gibi Weibull/log-normal karma sönümlü kanal modeli kullanılmıştır. Kablosuz iletişimde iletim ortamları olan, bina içi ve dışı çevreleri temsil etmede bu kanal modelinin kabiliyeti oldukça iyidir. Buna ilaveten, yine kablosuz iletişimde sıklıkla karşılaşılan çokyollu ve gölgeleme etkilerini yansıtmada başarılı olan iyi bir dağılımdır. Buna göre, bir karma Weibull/log-normal kanalının olasılık yoğunluk fonksiyonu (probability density function, PDF) şu şekilde bulunur:

$$
f(\gamma)=\int_{0}^{\infty} f(\gamma \mid x) f(x) d x .
$$


Burada $x$ alıcı taraftaki ortalama işaret-gürültü oranını (signal-to-noise ratio, SNR) belirtmekte iken $\gamma$ alınan anlık SNR'yi tanımlamaktadır. $f(\gamma \mid x)$ Weibull dağılımının PDF'sini, $f(x)$ ise log-normal dağılımının PDF'sini belirtmektedir ve sırasıyla şu şekildedir:

$$
\begin{aligned}
& f(\gamma \mid x)=\frac{c}{2}\left(\frac{\Gamma\left(1+\frac{2}{c}\right)}{x}\right)^{\frac{c}{2}} \gamma^{\frac{c}{2}-1} \exp \left(-\left(\frac{\gamma}{x} \Gamma\left(1+\frac{2}{c}\right)\right)^{\frac{c}{2}}\right), \gamma \geq 0 \\
& f(x)=\frac{1}{\sigma x \sqrt{2 \pi}} \exp \left(-\frac{\left(\log _{e} x-\mu\right)^{2}}{2 \sigma^{2}}\right) .
\end{aligned}
$$

burada $c$ Weibull sönümlenme parametresi, $\Gamma(\cdot)$ gamma fonksiyonunu belirtmektedir. $\mu$ ve $\sigma$ sirasiyla rastgele $\log _{e} x$ değişkeninin ortalama değerini ve standart sapmasını tanımlamaktadır. Eşitlik (2) ile verilen PDF ifadesi ters Weibull (inverse Weibull ya da Frechet) dağılımının genel bir halidir. Eşitlik (2) ve (3), (1)'de yerine yazılırsa ve [7, eşitlik (6)] yardımıyla bir karma Weibull/log-normal kanalının PDF'si

$$
f(\gamma) \approx \frac{2}{3} \varphi(\gamma ; \mu)+\frac{1}{6} \varphi(\gamma ; \mu+\sigma \sqrt{3})+\frac{1}{6} \varphi(\gamma ; \mu-\sigma \sqrt{3})
$$

olarak bulunur. Burada

$$
\varphi(\gamma ; y)=\frac{c}{2}\left(\frac{\Gamma\left(1+\frac{2}{c}\right)}{\exp (y)}\right)^{\frac{c}{2}} \gamma^{\frac{c}{2}-1} \exp \left(-\left(\frac{\gamma}{\exp (y)} \Gamma\left(1+\frac{2}{c}\right)\right)^{\frac{c}{2}}\right)
$$

şeklinde tanımlanmaktadır. Bir karma Weibull/log-normal kanalının CDF’sini bulmak için eşitlik (4) verilen PDF'nin integrali alınmalıdır. Buna göre, [7, eşitlik (8)] yardımıyla,

$$
F(\gamma) \approx \frac{2}{3}[1-\lambda(\gamma ; \mu)]+\frac{1}{6}[1-\lambda(\gamma ; \mu+\sigma \sqrt{3})]+\frac{1}{6}[1-\lambda(\gamma ; \mu-\sigma \sqrt{3})]
$$

şeklinde bulunur. Burada

$$
\lambda(\gamma ; y)=\exp \left(-\left(\frac{\gamma}{\exp (y)} \Gamma\left(1+\frac{2}{c}\right)\right)^{\frac{c}{2}}\right)
$$

olarak hesaplanmaktadır. Eşitlik (4) ile verilen bir karma Weibull/log-normal kanalın toplam ve yaklaşık PDF ifadesinin farklı Weibull parametrelerine (farklı $c$ değerlerine ait) göre değişimi Şekil 1'de verilmektedir. Eşitlik (6) ile elde edilen bir karma Weibull/log-normal kanalın toplam ve yaklaşı CDF ifadesinin farklı SNR değerlerine göre eşik SNR'ye bağlı değişim grafiği ise Şekil 2 ile gösterilmektedir.

Şekil 1 ve 2 ile verilen yaklaşı PDF ve CDF grafikleri gözönüne alındığında, eşitlik (4) ve (6) ile verilen yaklaşık PDF ve CDF ifadelerinin sonuçlarının gerçek (simülasyon) sonuçları ile oldukça uyumlu olduğu gözlenmiştir. Bu durum hata analizi sonunda elde edilecek olan kapalı formdaki nihai ifadenin doğruluğunu arttıracaktır. Çünkü kapalı formdaki hata olasılığı ifadesi, eşitlik (6) ile verilen yaklaşık CDF ifadesi kullanılarak bulunacaktır. 


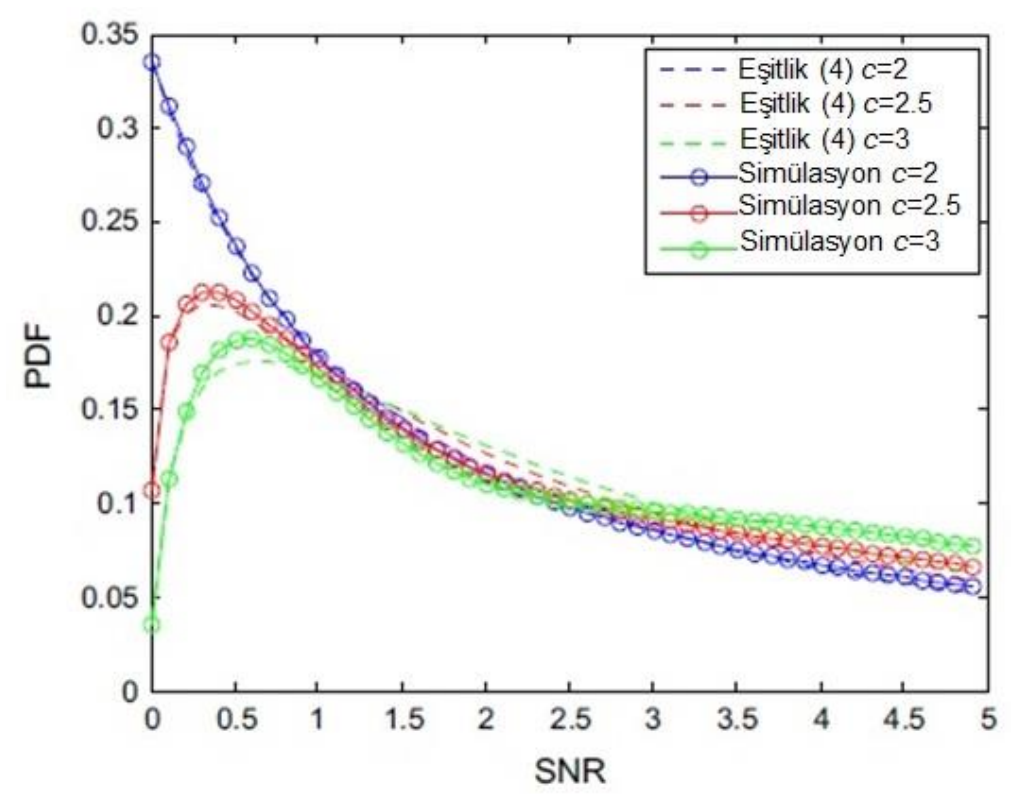

Şekil 1. Farklı Weibull sönümlenme parametrelerine göre eşitlik (4) ile verilen PDF ifadesinin nümerik ve simülasyon sonuçlarının klyaslanması [7]. (Comparison of numerical and simulation results for the PDF expression given by equation (4) with different Weibull fading parameters.)

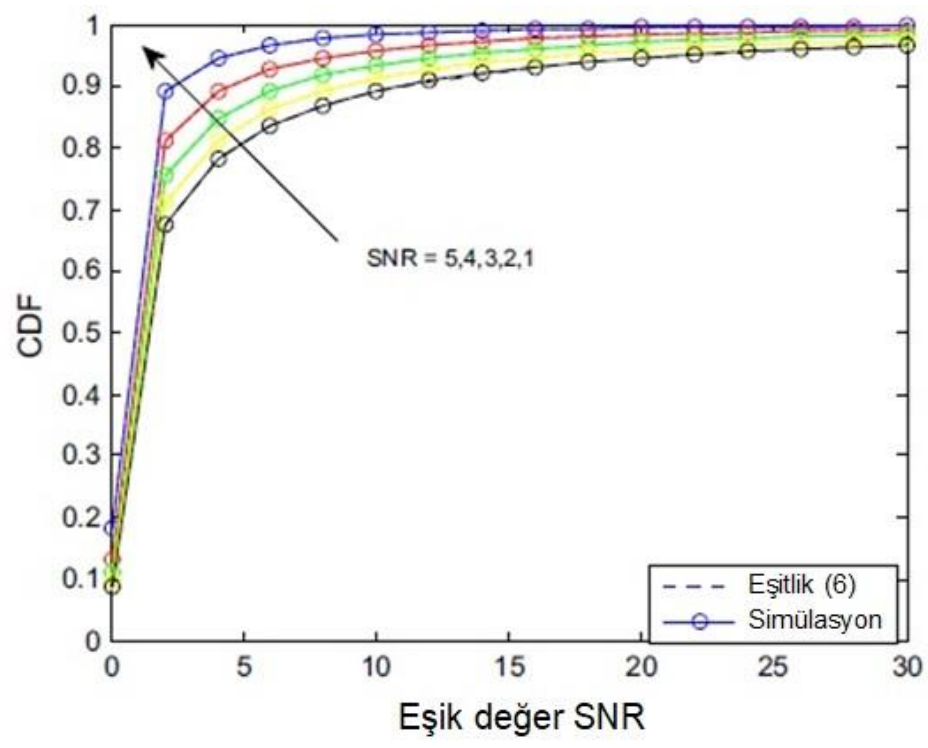

Şekil 2. Farklı SNR değerlerine göre eşitlik (6) ile verilen PDF ifadesinin nümerik ve simülasyon sonuçlarının klyaslanması [7]. (Comparison of numerical and simulation results for the CDF expression given by equation (6) with different SNR values.)

\section{HATA OLASILIĞI ANALIZİ (ERROR PROBABILITY ANALYSIS)}

Toplam CDF’ye bağlı olarak hata olasılı̆̆ı teorik olarak aşağıdaki gibi hesaplanır [21, eşitlik (20)]:

$$
P_{e}=\frac{r^{p}}{2 \Gamma(p)} \int_{0}^{\infty} \exp (-r \gamma) \gamma^{p-1} F(\gamma) d \gamma
$$


Burada $r$ ve $p$ farklı modülasyon türlerinin hesaplanması için kullanılan parametrelerdir. Örneğin; $r=1$, $p=0.5$ alındığında BPSK, $r=1, p=1$ alındığında DPSK ve $r=0.5, p=0.5$ alındığında BFSK olmaktadır. Eşitlik (6) ile bulunan toplam CDF ifadesi eşitlik (8)'de yerine yazılırsa

$$
\begin{gathered}
P_{e} \approx \frac{r^{p}}{2 \Gamma(p)} \int_{0}^{\infty} \exp (-r \gamma) \gamma^{p-1}\left\{\frac{2}{3}[1-\lambda(\gamma ; \mu)]+\frac{1}{6}[1-\lambda(\gamma ; \mu+\sigma \sqrt{3})]\right. \\
\left.+\frac{1}{6}[1-\lambda(\gamma ; \mu-\sigma \sqrt{3})]\right\} d \gamma
\end{gathered}
$$

şeklinde bir integral elde edilir. Bu integral, birbirine benzeyen 3 farklı integral çözümünden oluşmaktadır. Buna göre eşitlik (9) aşağıdaki gibi yeniden düzenlenebilir:

$$
P_{e} \approx \frac{r^{p}}{2 \Gamma(p)}\left\{I_{1}+I_{2}+I_{3}\right\}
$$

Burada $I_{1}$ integrali şu şekildedir:

$$
I_{1}=\int_{0}^{\infty} \exp (-r \gamma) \gamma^{p-1} \frac{2}{3}\left[1-\exp \left(-\gamma^{c / 2} \varepsilon_{1}^{c / 2}\right)\right] d \gamma
$$

Eşitlik (11) ile verilen integralde $\varepsilon_{1}=\Gamma(1+2 / c) / \exp (\mu)$ şeklinde tanımlanmaktadır ve bu integralde iki parçadan oluşmaktadır:

$$
\begin{aligned}
& I_{1}=\frac{2}{3}\left(Q_{1}-Q_{2}\right) \\
& Q_{1}=\int_{0}^{\infty} \exp (-r \gamma) \gamma^{p-1} d \gamma \\
& Q_{2}=\int_{0}^{\infty} \exp \left(-r \gamma-\gamma^{c / 2} \varepsilon_{1}^{c / 2}\right) \gamma^{p-1} d \gamma
\end{aligned}
$$

$Q_{1}$ integralinin çözümü [22, eşitlik (3.381.4)] yardımıyla şu şekilde türetilir:

$$
Q_{1}=\int_{0}^{\infty} \exp (-r \gamma) \gamma^{p-1} d \gamma=\frac{1}{r^{p}} \Gamma(p)
$$

$Q_{2}$ integralinin çözümü ise [23, eşitlik (2.3.2.13)] yardımıyla şu şekilde türetilir:

$$
\begin{aligned}
& Q_{2}=\int_{0}^{\infty} \exp \left(-r \gamma-\gamma^{c / 2} \varepsilon_{1}^{c / 2}\right) \gamma^{p-1} d \gamma \\
& Q_{2}=\left\{\begin{array}{l}
\sum_{j=0}^{q-1} \frac{\left(-\varepsilon_{1}^{c / 2}\right)^{j}}{j !} \Gamma\left(p+\frac{c}{2} j\right) r^{-p-\frac{c}{2} j}{ }_{t+1} F_{q}\left(1, \Delta\left(t, p+\frac{c}{2} j\right) ; \Delta(q, 1+j) ;(-1)^{q} z\right), 0<c<2 \\
\sum_{h=0}^{t-1} \frac{(-1)^{h}}{h ! c / 2} \Gamma\left(\frac{p+h}{c / 2}\right)\left(\varepsilon_{1}^{c / 2}\right)^{-(p+h) /(c / 2)}{ }_{q+1} F_{t}\left(1, \Delta\left(q, \frac{p+h}{c / 2}\right) ; \Delta(t, 1+h) ;(-1)^{t} z^{-1}\right), c>2
\end{array}\right.
\end{aligned}
$$

Burada $z=\left((t / r)^{t}\left(\varepsilon_{1}^{c / 2} / q\right)^{q}\right), \Delta(\cdot, \cdot)$ özel bir fonksiyon ve ${ }_{p} F_{q}(\cdot ; \cdot \cdot \cdot)$ genelleştirilmiş hipergeometrik fonksiyonunu ifade etmekte ve sırasıyla aşağıdaki gibi tanımlanmaktadır [24]: 


$$
\begin{aligned}
& \Delta(k, a)=\left(\frac{a}{k}, \frac{a+1}{k}, \ldots, \frac{a+k-1}{k}\right) \\
& { }_{p} F_{q}\left(a_{1}, \ldots, a_{p} ; b_{1}, \ldots, b_{q} ; x\right)=\sum_{k=0}^{\infty} \frac{\left(a_{1}\right)_{k}\left(a_{2}\right)_{k} \cdots\left(a_{p}\right)_{k}}{\left(b_{1}\right)_{k}\left(b_{2}\right)_{k} \cdots\left(b_{q}\right)_{k}} \frac{x^{k}}{k !}
\end{aligned}
$$

Eşitlik (14) ile verilen çözüm parçalı çözüm olup, $c=2$ durumunu içermemektedir. Bu sebeple, $c=2$ için çözüm ise [22, eşitlik (3.381.4)] yardımıyla

$$
Q_{2}=\int_{0}^{\infty} \exp \left(-r \gamma-\gamma \varepsilon_{1}^{c / 2}\right) \gamma^{p-1} d \gamma=\frac{1}{\left(r+\varepsilon_{1}^{c / 2}\right)^{p}} \Gamma(p)
$$

olarak elde edilir. Eşitlik (11)-(17) arası verilen çözüm işlemleri $I_{1}$ integrali içindir. Bu adımlara benzer şekilde $I_{2}$ ve $I_{3}$ integralleri de çözülebilir. Buna göre $I_{2}$ integrali için $\varepsilon_{2}=\Gamma(1+2 / c) / \exp (\mu+\sigma \sqrt{3})$ olarak tanımlanmakta ve

$$
\begin{gathered}
I_{2}=\frac{1}{6}\left(Q_{1}-Q_{3}\right) \\
Q_{3}=\int_{0}^{\infty} \exp \left(-r \gamma-\gamma^{c / 2} \varepsilon_{2}^{c / 2}\right) \gamma^{p-1} d \gamma \\
Q_{3}=\int_{0}^{\infty} \exp \left(-r \gamma-\gamma^{c / 2} \varepsilon_{2}^{c / 2}\right) \gamma^{p-1} d \gamma \\
Q_{3}=\left\{\begin{array}{l}
\sum_{j=0}^{q-1} \frac{\left(-\varepsilon_{2}^{c / 2}\right)^{j}}{j !} \Gamma\left(p+\frac{c}{2} j\right) r^{-p-\frac{c}{2} j}{ }_{t+1} F_{q}\left(1, \Delta\left(t, p+\frac{c}{2} j\right) ; \Delta(q, 1+j) ;(-1)^{q} z\right), 0<c<2 \\
\sum_{h=0}^{t-1} \frac{(-1)^{h}}{h ! c / 2} \Gamma\left(\frac{p+h}{c / 2}\right)\left(\varepsilon_{2}^{c / 2}\right)^{-(p+h) /(c / 2)}{ }_{q+1} F_{t}\left(1, \Delta\left(q, \frac{p+h}{c / 2}\right) ; \Delta(t, 1+h) ;(-1)^{t} z^{-1}\right), c>2
\end{array}\right.
\end{gathered}
$$

olarak çözümler elde edilmektedir. Aynı şekilde $I_{3}$ integrali için $\varepsilon_{3}=\Gamma(1+2 / c) / \exp (\mu-\sigma \sqrt{3})$ olarak alınır ve çözümü aşağıdaki gibi elde edilir:

$$
\begin{gathered}
I_{3}=\frac{1}{6}\left(Q_{1}-Q_{4}\right) \\
Q_{4}=\int_{0}^{\infty} \exp \left(-r \gamma-\gamma^{c / 2} \varepsilon_{3}^{c / 2}\right) \gamma^{p-1} d \gamma \\
Q_{4}=\int_{0}^{\infty} \exp \left(-r \gamma-\gamma^{c / 2} \varepsilon_{3}^{c / 2}\right) \gamma^{p-1} d \gamma \\
Q_{4}=\left\{\begin{array}{l}
\sum_{j=0}^{q-1} \frac{\left(-\varepsilon_{3}^{c / 2}\right)^{j}}{j !} \Gamma\left(p+\frac{c}{2} j\right) r^{-p-\frac{c}{2} j}{ }_{t+1} F_{q}\left(1, \Delta\left(t, p+\frac{c}{2} j\right) ; \Delta(q, 1+j) ;(-1)^{q} z\right), 0<c<2 \\
\sum_{h=0}^{t-1} \frac{(-1)^{h}}{h ! c / 2} \Gamma\left(\frac{p+h}{c / 2}\right)\left(\varepsilon_{3}^{c / 2}\right)^{-(p+h) /(c / 2)}{ }_{q+1} F_{t}\left(1, \Delta\left(q, \frac{p+h}{c / 2}\right) ; \Delta(t, 1+h) ;(-1)^{t} z^{-1}\right), c>2
\end{array}\right.
\end{gathered}
$$


$I_{1}, I_{2}$ ve $I_{3}$ integralleri için türetilen (13), (14), (17), (19) ve (21)'deki çözümler, eşitlik (10)'da yerine yazılarak bir karma Weibull/log-normal sönümlü kanal için hata olasılığı ifadesi kapalı formda elde edilir.

\section{NÜMERÍK SONUÇLAR (NUMERICAL RESULTS)}

Bu bölümde, bir karma Weibull/log-normal sönümlü kanaldaki SISO sisteme ait teorik hata olasıllı̆ı analizleri bilgisayar simülasyonlarıyla doğrulanmaktadır. Burada, BPSK, BFSK ve DPSK modülasyonlarına ait $c$ sönümlenme parametresinin farklı değerleri için hata performansı sonuçları verilmektedir. Ayrıca, BPSK ve DPSK modülasyon türlerini karşılaştırma amacıyla, farklı $c$ parametre değerlerine ele alacak şekilde SISO sisteminin hata performansı sonuçları da verilmektedir. Şekillerde, DPSK, BPSK ve BFSK modülasyonları için sırasıyla $(p, r)$ modülasyon sabitleri $(1,1),(0.5,1)$ ve $(0.5,0.5)$ olarak seçilmiştir. Şekil 3'te, DPSK modülasyonu kullanan SISO sisteminin farklı $c$ parametre değerleri için hata olasılığı performansları görülmektedir. Burada, $c$ sönümlenme parametresinin değiştirilmesi ile bir karma Weibull/log-normal sönümlü kanalın sönümlenme şiddetinin farklı olduğu durumlar gösterilmektedir.

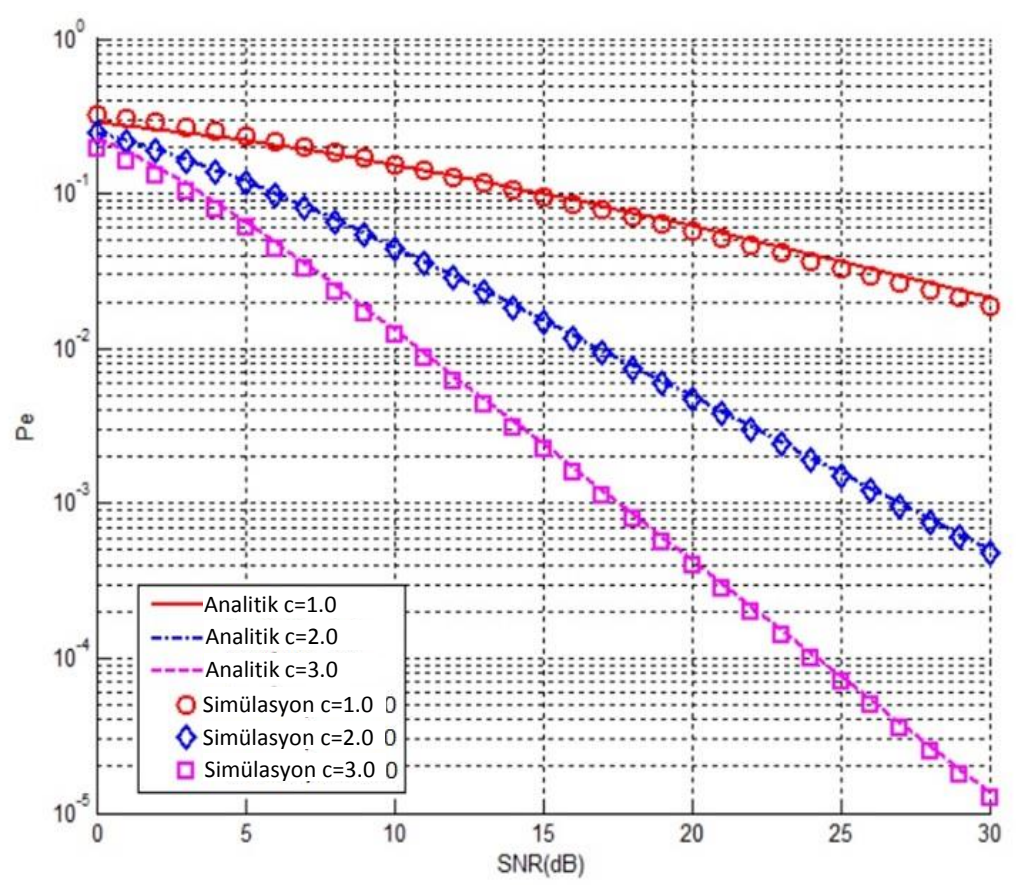

Şekil 3. DPSK modülasyonun kullanan SISO sistemin farklı c parametreleri için hata olasıllğ̆ (Error probability for different c parameters of the SISO system using DPSK modulation)

Şekil 3'teki eğriler, $c=1,2$ ve 3 için elde edilmiştir. Şekilden görüldüğü üzere simülasyon sonuçları ile teorik sonuçlar tam bir uyum içindedir. Sistem performansı $c$ sönümlenme parametresinin değeri arttıkça iyileşmektedir. Ayrıca, $c=1$ durumu için eşitlik (14), (19) ve (21)'in ilk kısımları $(0<c<2$ durumuna ait kısımlar), eşitlik (10)' da yerine yazılıp sonuca ulaşılmıştır. $c=3$ durumu için ise eşitlik (14), (19) ve (21)'in son kısımları ( $c>2$ durumuna ait kısımlar), eşitlik (10)'da yerine yazılıp hesaplanmıştır. $c=2$ durumunda ise eşitlik (17) kullanılarak, eşitlik (10) ifadesinden yararlanılmıştır. Hata olasılığı eğrilerinin eğimlerinden görüldüğü üzere, $c$ parametre değişimi performansı arttırmakta ve bilgisayar simülasyonlarıyla tutarlı sonuçlar vermektedir. $c$ sönümlenme parametresi 2 değerinden 3 değerine çıkarıldı̆̆ında, hata olasılı̆̆ının $10^{-2}$ değeri için, sistem performansı yaklaşık $7 \mathrm{~dB}$ iyileşmektedir.

Şekil 4'te, DPSK modülasyonu kullanan bir SISO sistem ile BPSK modülasyonu kullanan bir SISO sistemin bir Weibull/log-normal sönümlü kanalındaki hata olasılığı performansları karşılaştırılmaktadır. Şekil 4'teki eğriler, $c=2$ koşulları için elde edilmiştir. Şekilden görüldüğü gibi, (10)'da verilen yaklaşık hata olasılığı ifadesi kullanılarak elde edilen teorik sonuçlar ile simülasyon sonuçları oldukça uyumludur ve BPSK modülasyonunun üstünlüğü SNR değeri arttıkça ortaya çıkmaktadır. Hata olasılığının $10^{-3}$ değeri için, BPSK modülasyonu kullanan sistemin DPSK kullanan sisteme göre yaklaşık $3.5 \mathrm{~dB}$ daha iyi 
performansı sağlamaktadır. Şekil 5'te ise SISO sistemlerinde, karma Weibull/log-normal sönümlü kanalın sönümlenme parametre değerlerinin sirasıyla $c=1$ ve $c=2$ olduğunda ve farklı bir modülasyon türü olan BFSK modülasyonu kullanıldığında hata olasılığı performansına etkisi incelenmiştir. Beklendiği üzere, aynı sistem için sistemlerde $c$ değeri artırıldığında, sistemin hata olasıllğı performansı artmaktadır. Bu durum Şekil 5'te de gözlenmiştir. Sonuç olarak, kullanılan modülasyona bağlı olmaksızın sönümlenme parametresinin artması, karma Weibull/log-normal sönümlü kanalın iyileşmesine neden olmakta ve bu sayede hata performansı iyileşmektedir.

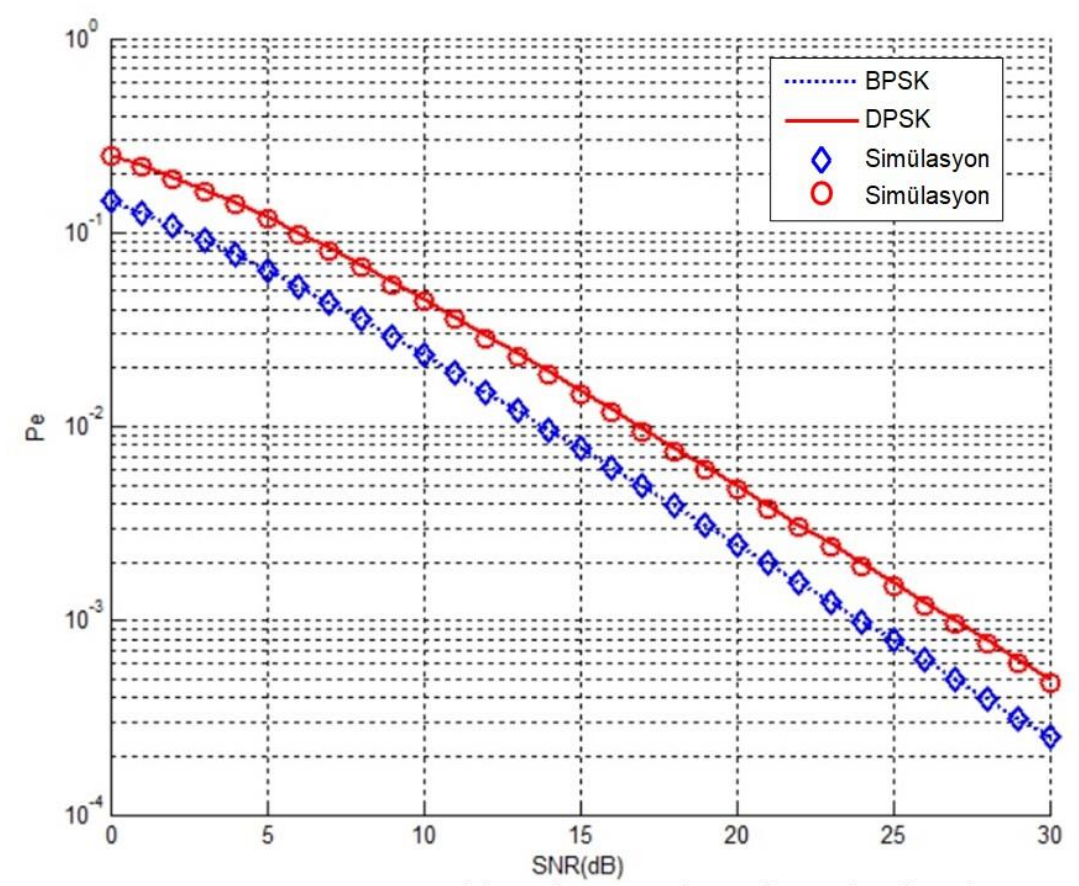

Şekil 4. $c=2$ için BPSK ve DPSK modülasyonlarının performanslarının karşıllaştırılması (Comparison of $B P S K$ and DPSK modulation performances of our system for $c=2$ )

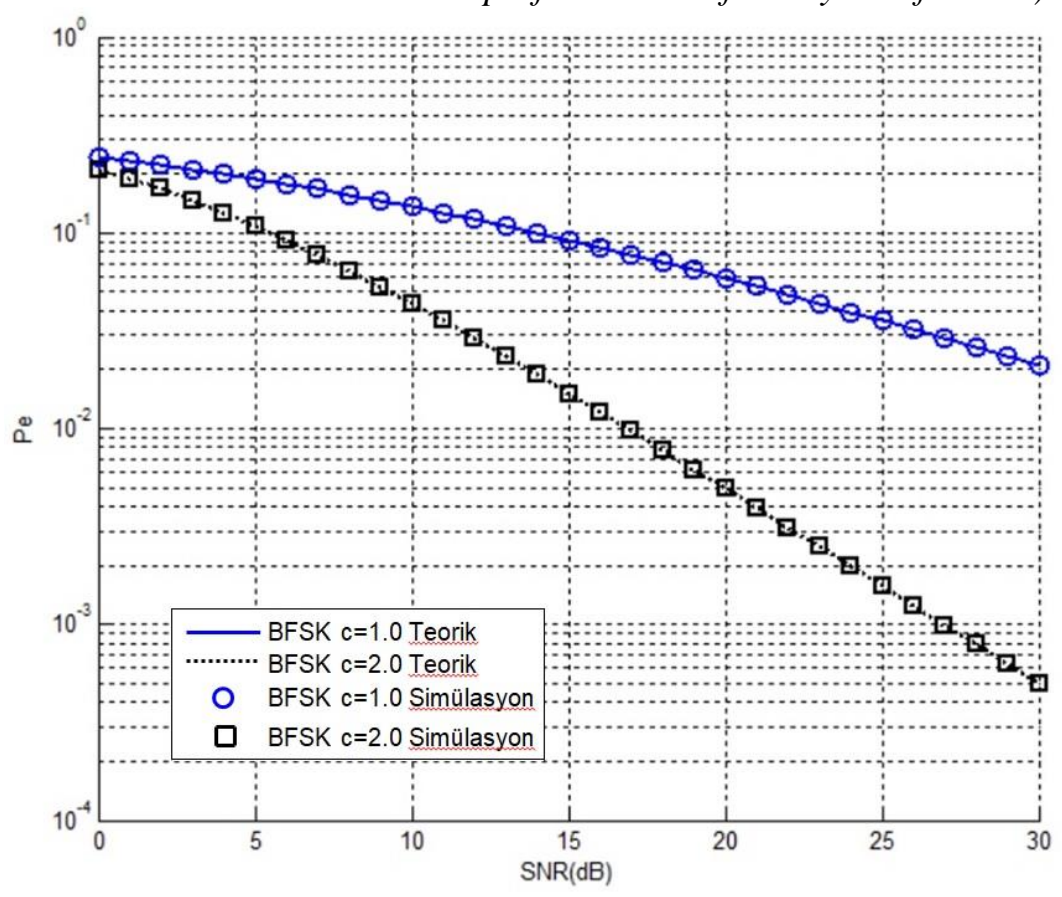

Şekil 5. BFSK modülasyonu kullanan SISO sistemi için $c=1$ ve $c=2$ durumlarının hata olasıllı̆ karşılaştırmast. (Comparison of $c=1$ and $c=2$ conditions for error probability of SISO system using BFSK modulation) 


\section{SONUÇ (CONCLUSION)}

Bu çalışmada bir SISO sisteminin karma Weibull/log-normal sönümlü kanallardaki hata olasılığı analizi hem teorik hem de simülasyonlar aracıllğı ile yapılmıştır. Sunulan teorik analiz gerçekleştirilirken sisteme ait toplam PDF ifadesi bulunmuş, elde edilen PDF yardımı ile sisteme ait toplam yaklaşı CDF ifadesi elde edilip, bu ifade kullanılmıştır. Sistemin hata olasılığı üzerindeki etkileri farklı kanal sönümlenme değerleri için detaylı bir şekilde analiz edilmiştir. Buna ek olarak ele alınan sistemin performans analizleri farklı modülasyon türleri göz önünde bulundurularak çeşitli senaryolar ile araştırılmıştır. Elde edilen sonuçlara göre teorik ve simülasyon sonuçları oldukça uyumlu iken, sönümlenme parametresi değeri arttıkça performansın beklendiği üzere iyileştiği görülmüştür.

\section{KAYNAKLAR (REFERENCES)}

[1]. Fodor G., Rajatheva N., Zirwas W., Thiele L., Kurras M., Guo K., Tölli A., Sorensen J.H., de Carvalho E., "An overview of massive MIMO technology components in METIS", IEEE Commun. Mag, Cilt 55, No 6, 155-161, 2017.

[2]. Xu C., Hu Y., Liang C., Ma J., Ping L., "Massive MIMO, non-orthogonal multiple access and interleave division multiple access", IEEE Access, Cilt 5, 14728-14748, 2017.

[3]. Shafi M., Molisch A.F., Smith P.J., Haustein T., Zhu P., De Silva P., Tufvesson F., Benjebbour A., Wunder G., "5G: a tutorial overview of standards, trials, challenges, deployment, and practice", IEEE J. Sel. Areas Commun., Cilt 35, No 6, 1201-1221, 2017.

[4]. Sonmez M., "PDSM sinyalinin çoklu örnekleme tekniği kullanılarak demodülasyonu," GU J Sci Part C., Cilt 5, No 4, 145-150, 2017.

[5]. Lu L., Li G.Y., Swindlehurst A.L., Ashikhmin A., Zhang R., "An overview of massive MIMO: benefits and challenges," IEEE J. Sel. Topics Signal Process., Cilt 8, No 5, 742-758, 2014.

[6]. Nakagami, M. "The $m$-distribution, a general formula of intensity distribution of rapid fading", Oxford: Pergamon, England, 1960.

[7]. Singh R., Soni S.K., Raw R.S., Kumar S., "A new approximate closed-form distribution and performance analysis of a composite Weibull/Log-Normal fading channel", Wireless Pers. Commun., Cilt 92, No 3, 883-890, 2017.

[8]. Yacoub M.D., "The $\kappa-\mu$ distribution and the $\eta-\mu$ distribution”, IEEE Antennas Propag. Mag., Cilt 49, No 1, 68-81, 2007.

[9]. Paris J.F., "Statistical characterization of $\kappa-\mu$ shadowed fading", IEEE Trans. Veh. Technol., Cilt 63, No 2, 518-526, 2014.

[10]. Yacoub M.D., “The $\alpha-\mu$ distribution: A physical fading model for the Stacy distribution”, IEEE Trans. Veh. Technol., Cilt 56, No 1, 27-34, 2007.

[11]. Peña-Martín J.P., Romero-Jerez J.M., Tellez-Labao C., "Performance of selection combining diversity in $\eta-\mu$ fading channels with integer values of $\mu$ ", IEEE Trans. Veh. Technol., Cilt 64, No 2, 834-839, 2015.

[12]. Villavicencio M.A.G., de Souza R.A.A., de Souza G.C., Yacoub M.D., “A bivariate $\kappa-\mu$ distribution”, IEEE Trans. Veh. Technol., Cilt 65, No 7, 5737-5743, 2016.

[13]. Yacoub M.D., "The $\alpha-\eta-\kappa-\mu$ fading model”, IEEE Trans. Veh. Technol., Cilt 64, No 8, 3597-3610, 2016.

[14]. Hashemi, H. "The indoor radio propagation channel”, Proceedings of IEEE, Cilt 81, No 7, $943-$ 968, 1993.

[15]. Tzeremes G., Christodoulou C.G., "Use of Weibull distribution for describing outdoor multipath fading”, IEEE Antennas and Propagation Society International Symposium, San Antonio, Texas, USA, 232-235, 16-21 Haziran 2002.

[16]. Babich F., Lombardi G., "Statistical analysis and characterization of the indoor propagation channel", IEEE Trans. Commun., Cilt 48, No 3, 455-464, 2000.

[17]. Bilim M., Kapucu N. Develi I., "Dual-hop $N$-relay assisted transmission for IDMA systems over Weibull fading channels", Elektronika Ir Elektroniteknika, Cilt 21, No 4, 69-72, 2015. 
[18]. Bilim M., Kapucu N. Develi I., "Cooperative IDMA systems with regenerative relays over Weibull fading channels: outage probability and error analysis", IET Commun., Cilt 11, No 7, 993-999, 2017.

[19]. Adawi N., Bertoni H.L., Child J.R., "Coverage prediction for mobile radio systems operating in the 800/900 MHz frequency range”, IEEE Trans. Veh. Technol., Cilt 37, No 1, 3-72, 1988.

[20]. Mitic, M.A., Jakovljevic, M.M., "Second-order statistics in Weibull-lognormal fading channels", In: Proceedings of International Conference on Telecommunications in Modern Satellite, Cable and Broadcasting Services, Serbia, 529-532, 26-28 Eylül 2007.

[21]. Sharma N., Bansal A., Garg P., "Decode-and-forward relaying in mixed $\eta-\mu$ and gamma-gamma dual hop transmission system", IET Commun., Cilt 10, No 14, 1769-1776, 2016.

[22]. Gradshteyn, I. S., Rzyhik, I. M., "Table of integrals, series and products", Academic Press, London, UK, 2007.

[23]. Prudnikov, A. P., Brychkov,Y. A., Marichev,O. I.. "Integrals and series (Vol. 1)”, London: Gordon, UK, 1986.

[24]. Nadarajah S., Kotz S., "On the Weibull MGF”, IEEE Trans. Commun., Cilt 55, No 7, 1287, 2007. J. Bear, Hydraulics of Groundwater, Dover, Minneola, 2007. 